innate and adaptive immune responses. High numbers of IL-17producing $\mathrm{T}$ cells which also secrete IL-21 and IL-22 are found in close proximity to bile ducts in several liver diseases. $\mathrm{T}_{\mathrm{h} 17}$ related cytokines have multiple effects and may be involved in both effector responses and repair and regeneration.

Methods Primary human parenchymal cells were assessed for cytokine receptor expression by western blotting. The effects of stimulation with recombinant IL-17, IL-21, IL-22, TNFa or IFN-g alone or in combination were compared for apoptosis using annexin staining, proliferation was measured by in situ Ki67 staining and adhesion molecule expression was assessed by flow cytometry. Secretion of IL-1b, IL-6, IL-23 and TGF-b1 was assessed by ELISA. Results All parenchymal cells expressed IL-17R, IL-21R and IL-22R. $\mathrm{T}_{\mathrm{h} 17}$ related cytokines did not cause apoptosis but led to parenchymal cell proliferation. Cholangiocytes and hepatocytes responded best to IL-17, whereas sinusoidal endothelial cells were responsive to IL-22. Endothelial cells upregulated adhesion molecules in response to $\mathrm{T}_{\mathrm{h} 17}$ related cytokines. Cholangiocytes responded to $T_{h 17}$ cytokines by secreting high levels of IL-1b, IL-6, IL-23 and TGF-b1 all cytokines that support the survival of $\mathrm{T}_{\mathrm{h} 17}$ and $\mathrm{T}_{\mathrm{c} 17}$ cells.

Conclusion Liver parenchymal cells express IL-17, IL-21 and IL-22 receptors and proliferate in response to $T_{h 17}$ cytokines. Upregulation of adhesion molecules by sinusoidal endothelial cells promotes lymphocyte recruitment and retention. Cholangiocytes also respond by secreting $T_{h 17} / T_{c 17}$ polarising cytokines. Therefore $T_{h 17}$ related cytokines secreted by infiltrating lymphocytes may activate the epitheliome to generate a local environment characterised by cholangiocyte proliferation and $T_{h 17} / T_{c 17}$ cell survival, thus contributing to bile duct proliferation and persistent chronic inflammation that characterises many liver diseases.

Competing interests None declared.

\section{PM0-119 PHENOTYPICALLY AND FUNCTIONALLY DISTINCT MONOCYTE SUBSETS AND THEIR ROLE IN HUMAN LIVER DISEASE}

doi:10.1136/gutjnl-2012-302514b.119

${ }^{1}$ E Liaskou, ${ }^{1,2} \mathrm{H}$ Zimmermann, ${ }^{1} Z$ Stamataki, ${ }^{3} 0$ Qureshi, ${ }^{4} \mathrm{~S} S$ Choi, ${ }^{1 J}$ Shaw, 'S M Curbishley, ${ }^{1,5,6}$ W K Syn, 'D H Adams. ${ }^{1}$ Centre for Liver Research and NIHR Biomedical Research Unit in Liver Disease, Institution of Biomedical Research, University of Birmingham, Birmingham, UK; ${ }^{2}$ Medical Department III, University Hospital of Aachen, Aachen, Germany; ${ }^{3}$ Medical Research Council (MRC) Centre for Immune Regulation, School of Immunity and Infection, Institute of Biomedical Research, University of Birmingham Medical School, Birmingham, UK; ${ }^{4}$ Division of Gastroenterology, Department of Medicine, Duke University, Durham, North Carolina, USA; ${ }^{5}$ The Institute of Hepatology, Regeneration and Repair Group, London, UK; ' ${ }^{6}$ Department of Physiology, University of the Basque Country, Bilbao, Spain

Introduction Chronic liver inflammation is a leading cause of morbidity and mortality world wide, characterised by a dysregulated tissue repair driven by uncontrolled inflammation that leads to fibrosis, cirrhosis and hepatocellular carcinoma. We have investigated the role of different monocyte subsets: classical (CD14+ +CD16-/Mon1), intermediate (CD14++CD16+/Mon2) and nonclassical (CD14+CD16++/Mon3) monocytes in human liver disease.

Methods Liver-infiltrating and peripheral blood mononuclear cells (MNC) were isolated from normal individuals or patients with liver disease (ALD/NASH/PSC/AIH) and sorted into phenotypic subsets that were studied for their differentiation in response to Th1/Th2 cytokines by flow cytometry, migration across TNF $\alpha / \mathrm{IFN} \gamma$ stimulated hepatic sinusoidal endothelial cells (HSEC) under physiological flow and phagocytic activity of zymosan bioparticles. The ability of different monocytes to activate hepatic stellate cells (HSC) was assessed using ORT-PCR (aSMA and COL1 11 gene expression changes). In this study monocyte subsets are defined as Mon1 $(\mathrm{CD} 14++\mathrm{CD} 16-)$, Mon2 (CD14++CD16++) and Mon3 (CD14 $+\mathrm{CD} 16++)$. In functional experiments Mon2 and Mon3 were studied together and are defined as total CD16+ monocytes.

Results Mon1 comprised $80 \%$ of MNC in the blood of healthy subjects and patients with liver disease but only $50 \%$ of MNC in both normal and diseased liver. Mon2 comprised 9\% and 14\% of $\mathrm{MNC}$ in normal and diseased blood respectively, but were significantly increased in normal and diseased livers (42 and 30\% of MNC, respectively). Transmigration of total $\mathrm{CD} 16+$ monocytes across inflamed HSEC was 2.3-fold higher compared to CD14+ respectively. In vitro stimulation of Mon1 with TGF $\beta 1$ or IL-10 for 5 days induced 16- and 20-fold increases in CD16 expression. Liver infiltrating Mon2 expressed higher levels of CD163 and HLA-DR compared to Mon1, colocalized with CD68 and demonstrated high phagocytic activity, indicative of a macrophage phenotype. Diseased-liver-derived total CD16+ monocytes secreted higher levels of CCL2, IL-6, IL-8 and IL-13 and induced a twofold increase in aSMA and COL1a1 expression in co-cultured HSC.

Conclusion Compared with normal livers, diseased livers harbour fewer CD14+ but significantly more CD16+ monocytes that secrete profibrotic cytokines and are able to activate HSC. CD16+ monocyte accumulation in the liver is the result of enhanced recruitment from blood and also local differentiation from CD14+ in response to TGF $\beta 1$ and IL-10 present in the fibrotic microenvironment.

Competing interests None declared.

\section{PM0-120 KUPFFER CELL DERIVED INTERLEUKIN (IL)-18 INDUCES HEPATIC INFLAMMATION BY PROMOTING LYMPHOCYTE SUBSETS RECRUITMENT ON HEPATIC ENDOTHELIAL CELLS}

doi:10.1136/gutjnl-2012-302514b.120

${ }^{1} E$ Liaskou, ${ }^{*} \mathrm{D}$ Withers, ${ }^{1} E$ Humphreys, ${ }^{1} \mathrm{~J} C$ Shaw, ${ }^{1} \mathrm{~L}$ Tuohey, ${ }^{3} \mathrm{P}$ Klenerman, ${ }^{1} \mathrm{D}$ H Adams, ${ }^{1} \mathrm{Y}$ H Oo. ${ }^{1}$ Division of Immunity and Infection, Centre for Liver Research \& NIHR BRU, UK; ${ }^{2}$ MRC Centre for Immune Regulation, University of Birmingham, Birmingham, UK; ${ }^{3}$ Peter Medawar Building for Pathogen Research, University of Oxford, Oxford, UK

Introduction IL-18, known as interferon- $\gamma$ inducing factor, is a potent pro-inflammatory cytokine implicated in liver allograft rejection, viral hepatitis and hepatocellular carcinoma progression, where it plays an important role in cell-mediated immune responses and inflammatory injury. We hypothesise that IL-18 promotes hepatic inflammation by supporting effector T cells migration across hepatic sinusoidal endothelium. In this study, we investigated the expression and cellular regulation of IL-18 secretion in the human liver and demonstrated a role in promoting $T$ cell recruitment to the liver.

Methods IL-18 mRNA expression levels were measured in normal and diseased human livers using ORT-PCR and tissue localisation assessed by immunohistochemistry and confocal microscopy. Human hepatic sinusoidal endothelial cells were treated with Il-18 in vitro and flow cytometry used to assess induction of adhesion molecules. The functional significance of these responses to IL-18 was investigated in flow based adhesion assays using IL-18 treated HSEC and CD4 and CD8 T cell subsets under physiological flow.

Results IL-18 mRNA expression was significantly higher in liver tissue form patients with ALD (19-fold), PBC (7.6-fold) and seronegative hepatitis $(30.6$-fold) $(\mathrm{p}<0.05)$ compared with normal liver. IL-18 protein expression was restricted to hepatic sinusoids where it colocalized with $\mathrm{CD}^{+} 8^{+}$Kupffer cells, whereas CD31 ${ }^{+}$endothelial cells were IL-18 ${ }^{\text {neg }}$. HSEC stimulated with IL-18 lead upregulation of cell adhesion molecules ICAM- 1 and VCAM- 1 which translated into a 2.5-fold increase in their functional ability to recruit CD4 and CD8 
T cells in flow-based adhesion assays. Total adhesion of CD4 and CD8 T cells was significantly $(p<0.05)$ reduced when ICAM- 1 , VCAM-1, and CXCR3 molecules were blocked or if G protein coupled receptors were inhibited with pertussis toxin (PTX). CD8 T cell adhesion was also dependent on vascular adhesion protein-1.

Conclusion We report high IL-18 expression by Kupffer cells in inflammatory liver disease. The ability of IL-18 to enhance T cell recruitment via sinusoidal endothelium suggests it acts to promote lymphocyte recruitment during the development of chronic hepatitis and is thus a potential novel therapeutic target in inflammatory liver disease.

Competing interests None declared.

\section{PMO-121 OSTEOPONTIN PROMOTES NATURAL KILLER T (NKT) CELL ACCUMULATION IN NONALCOHOLIC STEATOHEPATITIS (NASH)}

doi:10.1136/gutjnl-2012-302514b.121

${ }^{1} \mathrm{E}$ Liaskou, ${ }^{* 1} \mathrm{~L} \mathrm{C}$ Claridge, ${ }^{1,2} \mathrm{R}$ Younis, ${ }^{1} \mathrm{H}$ Shah, ${ }^{1} \mathrm{~J}$ Shaw, ${ }^{1} \mathrm{Y} \mathrm{H}$ Oo, ${ }^{3} \mathrm{Z} \mathrm{Mi},{ }^{3} \mathrm{P} \mathrm{C}$ Kuo, ${ }^{4} \mathrm{~A}$ Canbay, ${ }^{1} \mathrm{D}$ H Adams, ${ }^{5,6} \mathrm{~A}$ M Diehl, ${ }^{1,2} \mathrm{~W}$ K Syn. ${ }^{1}$ Centre for Liver Research, University of Birmingham, Birmingham, UK; ${ }^{2}$ Regeneration and Repair, Institute of Hepatology, London, UK; ${ }^{3}$ Department of Surgery, Loyola University, Chicago, USA; ${ }^{4}$ Department of Gastroenterology, Essen University, Essen, Germany; ${ }^{5}$ Department of Medicine, Duke University, Durham, USA; ${ }^{6}$ Department of Gastroenterology, Duke University, Durham, USA

Introduction Progressive steatohepatitis is characterised by increased inflammatory cell infiltration. Osteopontin (OPN) is a cytokine intricately associated with immune-cell accumulation, and we reported that NKT cells accumulate to promote hepatic injury in NASH. We hypothesise that OPN promotes NASH progression by supporting NKT migration across hepatic sinusoidal endothelium. Methods Mice were fed chow or methionine-choline deficient (MCD) diet to induce NASH. After 4 weeks, mice were sacrificed; severity of disease assessed by serum aminotransferase (AST), liver OPN quantified by ORTPCR and immunohistochemistry, blood OPN measured by ELISA. In separate studies, MCD-fed mice were treated with sham or OPN aptamers, and FACS used to quantify liver NKTs. Primary human hepatic sinusoidal endothelial cells (HSEC) were stimulated with recombinant (r)OPN (0-1000 ng/ $\mathrm{ml})$, with or without TNFa $(20 \mathrm{ng} / \mathrm{ml})+\operatorname{IFNg}(100 \mathrm{ng} / \mathrm{ml})$, and expression of adhesion molecules (ICAM1, VCAM1) and chemokines (CXCL9, 10, 11, 16) assessed. To assess lymphocyte migration, lymphocytes were perfused over rOPN-or vehicle-treated-HSEC, with or without TNFa+IFNg. In separate experiments, TNFa + IFNg stimulated-HSEC were treated with OPN aptamers or blocking antibodies, and total lymphocyte adhesion recorded. Human NASH livers were immunostained for OPN, plasma measured for OPN, and liver NKT numbers from normal or NASHcirrhotic patients quantified by FACS.

Results In mice, MCD-induced NASH upregulated expression of liver OPN by threefolds $(p<0.05)$, blood OPN by twofolds $(p<0.05)$, increased liver CD4 by 2.2-fold, and NKT cells by threefold $(p<0.05)$. $\mathrm{MCD}$-fed mice treated with OPN aptamers accumulated fewer CD4 and NKT cells $(p<0.05)$, and exhibited attenuated injury (ALT: twofold reduction; $\mathrm{p}<0.02)$. rOPN induced expression of ICAM-1, VCAM-1 and CD31 on human HSEC, enhanced lymphocyte recruitment under conditions of flow (40\%), and amplified recruitment ability of TNFa+IFNg stimulated HSEC (20\%), while OPN neutralisation with RNA-aptamers reduced lymphocyte recruitment by $50 \%($ all $p<0.05)$. OPN effects were mediated via CD $44, a_{v} b_{3}$, ICAM1, VCAM1, and CXCL16. In humans, liver and plasma OPN was significantly upregulated in NASH; livers from NASH-cirrhosis harboured twofolds more CD4 and threefolds more NKT cells $(p<0.05)$ than normal.

Conclusion Liver and plasma OPN levels are upregulated during steatohepatitis in mice and humans, and promote liver NKT accumulation. OPN neutralisation significantly reduces lymphocyte subset recruitment and liver injury, suggesting that OPN is a promising anti-inflammatory target in steatohepatitis.

Competing interests None declared.

\section{PM0-122 EICOSAPENTAENOIC ACID IS EFFECTIVE AT REDUCING HEPATOCYTE TRIGLYCERIDE CONTENT OF UNTREATED C3A CELLS BUT IS NOT EFFECTIVE IN TWO MODELS OF CELLULAR STEATOSIS}

doi:10.1136/gutjnl-2012-302514b.122

G Masterton, * P Cowan, P Lee, E Pryde, P C Hayes, J N Plevris. Division of Clinical and Surgical Sciences, University of Edinburgh, Edinburgh, UK

Introduction Eicosapentaenoic acid (EPA), one of the major physiologically active constituents of Omega- 3 fatty acids, has been suggested as a treatment for non-alcoholic fatty liver disease (NAFLD). The aim of these experiments was to assess the effects of EPA on intrahepatic triglyceride content in cell culture models of steatosis.

Methods Human C3a hepatocytes were incubated in MEME (standard media) and two models of cellular steatosis: oleate (a model of isolated steatosis) and LPON (a model of steatosis and mitochondrial dysfunction containing the gluconeogenic substrates Lacate, Pyruvate, Octanoate and ammoNia). Test media was either unsupplemented, or supplemented with $50 \mu \mathrm{M}$ or $250 \mu \mathrm{M}$ EPA. Hepatocyte triglyceride accumulation was assessed both by microscopy (using oil red staining) and by quantifying the intracellular triglyceride concentration of cells incubated in culture media for 3 and 7 days. Each cell culture experiment was performed in triplicate.

Results $\boldsymbol{M E M E}$ When quantified by oil red staining a $73.1 \%(95 \% \mathrm{CI}$ $63 \%$ to $83 \%$ ) reduction in cell triglyceride content with $250 \mu \mathrm{M} \mathrm{EPA}$ compared with untreated cells was seen (7659 vs 28564 pixels; $\mathrm{p}<0.001)$. This was confirmed in cell culture experiments as $250 \mu \mathrm{M}$ EPA was associated with reduced intrahepatocyte triglyceride content after both 3 (74.1 vs $94.9 \mathrm{mmol} / \mathrm{gTP} ; \mathrm{p}<0.05)$ and 7 days (62.2 vs $80.9 \mathrm{mmol} / \mathrm{gTP} ; \mathrm{p}<0.05)$ incubation compared with untreated cells equating to a $21.9 \%$ (95\% CI $9 \%$ to $35 \%$ ) and $23.1 \%$ (95\% CI $5 \%$ to $41 \%)$ reduction respectively. For both experiments a linear trend between increasing EPA concentration and reduced triglyceride content was confirmed. Oleate Here reduced triglyceride content with both $50 \mu \mathrm{M}$ EPA $(\mathrm{p}<0.01)$ and $250 \mu \mathrm{M}$ EPA $(\mathrm{p}<0.05)$ was seen with oil red staining and equated to reductions of $27.6 \%$ (95\% CI $16 \%$ to $39 \%$ ) and $22.5 \%$ (95\% CI $9 \%$ to $36 \%$ ) compared with untreated cells. However these results were not reproduced in cell culture experiments although on post test analysis there was a significant linear trend between increasing EPA concentration and reduced triglyceride content $(p=0.04)$. LPON Although incubation with $250 \mu \mathrm{M}$ EPA reduced triglyceride content in the LPON model when quantified with oil red staining (60308 vs 79219 pixels in $250 \mu \mathrm{M} \mathrm{EPA}$ vs untreated cells; $p<0.05)$ this was not confirmed in cell culture experiments. On post hoc analysis no trend was demonstrated between EPA concentration and triglyceride content.

Conclusion These results suggest that EPA is effective at reducing triglyceride accumulation in untreated hepatocytes but is not effective in either oleate or LPON models of cellular steatosis. It is therefore possible that the presence of steatosis and mitochondrial dysfunction in NAFLD may limit the efficacy of EPA as a treatment.

Competing interests None declared. 